\title{
Preclinical Screening of Anticancer Drugs using Infrared (IR) Micro-spectroscopy
}

\author{
Caryn Hughes $^{1,2^{*}}$, Graeme Clemens $^{2,3}$ and Matthew J. Baker ${ }^{2 *}$ \\ ${ }^{1}$ University of Manchester, Brunswick Street, Manchester, M13 9PL, UK \\ ${ }^{2}$ WestCHEM, Department of Pure and Applied Chemistry, University of Strathclyde, 295 Cathedral Street, \\ Glasgow, G1 1XL, UK \\ ${ }^{3}$ Centre for Materials Science, Division of Chemistry, School of Forensic and Investigative Sciences, University \\ of Central Lancashire (UCLan), Preston, PR1 2HE, UK \\ *caryn.hughes@manchester.ac.uk, matthew.baker@strath.ac.uk \\ Twitter @Dr_Caryn_Hughes, @ChemistryBaker
}

Keywords: cancer, infrared, spectroscopy, high-throughput, preclinical screening, biomarker

Research and development costs associated with successful market release of anticancer therapeutics are notoriously high. Whilst traditional drug screening approaches have served the cancer research community well over the last few decades [1], drug discovery programs are protracted, driving a continuous demand to develop non-traditional preclinical tools that are accurate, high throughput and cost-effective.

Fourier Transform Infrared micro-spectroscopy (FTIRM) can provide an operational platform for such a strategic approach. The method involves a non-destructive and label-free process of analysis that enables subsequent histological reporting. The technique provides a unique fingerprint spectrum containing global biochemical information of the main macromolecules that compose a cell; either at the single cell level, or as an overall signal from a global cell population.

Evidence of drug-cell interaction and drug efficacy can be inferred by the acquisition of infrared spectral data from drug-treated cells (Figure 1). It is possible to detect discriminatory changes to the cellular IR signal even before visible morphological changes are evident, producing data of diagnostic significance for drug screening applications. Interestingly, an IR cell apoptosis-specific marker was identified and corroborated with flow cytometry over a decade ago [2]. Progress of the method for preclinical application, however, has been hampered; physical spectral distortion phenomena and data processing hurdles have resulted in concerns about data misinterpretation. Recently, however, many of these issues have now been addressed by advances in the theoretical understanding underpinning the spectral acquisition process [3]. Coupled with the internationally-strong biospectroscopy community driving to establish robust and standardized methodologies in order to improve inter lab repeatability, FT-IRMS is beginning to show its true potential [4].

As a result, cellular responses to therapeutic agents can now be studied with FTIRM in confidence. The technique has high potential as a preclinical tool in the early drug discovery process in terms of identifying lead compounds and weeding out weak candidates. In addition to determining apoptotic signals, knowledge of the IR spectral changes associated with inherent cell cycle chemistry has also improved. This will undoubtedly enhance information gained when distinguishing spectral signals of normal cellular events with cellular responses to cycle-arresting drugs. Detection and classification of growth phases of individual cells based on their label-free IR spectra has been demonstrated to be 
achievable on both a chemically-fixed [5] and live-cell timescale [6] thus, highlighting its diverseness. Furthermore, as demonstrated in FTIRM studies of multiple cell lines, it is possible to achieve general classification of drug mode of action by the cellular response [7]. As a preclinical tool, this provides significant benefit for the analysis of experimental compounds with unknown chemical mechanisms.

In addition to pre-clinical screening, there are clinical applications for using an IR based tool that will result in beneficial patient impact. For instance, evidence gathered thus far has indicated that IRbased detection of a cellular response to sub-toxic doses is possible [8] and efforts are also being directed to the study of IR spectral biomarkers for cellular sensitivity and resistance [9]. Close monitoring of IR spectral changes in such markers, used in combination with other analytical methods, could provide fundamental indications of favourable patient responders to certain drugs for exploitation in personalised medicine.

The majority of insights gained from drug-cell interaction studies on the cellular level in previous years have been discovered using synchrotron-FTIRM due to their brilliant sources. This is because the IR light had to be confined to small sample areas in order to probe a single biological cell. With such constraints imposed on the light signal, conventional benchtop IR systems with a lowthroughput thermal source struggled to achieve an effective signal to noise ratio (SNR). Conversely, the use of a high-intensity synchrotron light source ensured that the SNR was uncompromised.

The use of synchrotron radiation may be advantageous for specialised, focused experiments, however their use for the application of IR preclinical screening tool it is an impractical notion. Fortuitously, the field has benefited from a recent surge in technological advance. Benchtop IR systems with the standard thermal sources can now achieve comparable SNR at the single cell level due to the advent of $128 \times 128$ element focal plane array detectors [10]; an infrared spectrum can be recorded on a pixel by pixel basis, sampling a large area of multiple cells in a spatially-resolved chemical map. The SNR can be increased by the identification and sub sequential averaging of multiple spectra that are associated with an individual single cell. Jimenez-Hernandez et al [5] demonstrated this approach and was able to sample multiple cells 10-20 $\mu \mathrm{m}$ in diameter within a $700 \times 700 \mu \mathrm{m}$ area simultaneously. Until very recently, such benchtop FTIR imaging studies have been performed at pixel resolution of $5.5 \mu \mathrm{m}$ - far behind the diffraction-limited pixel resolution of $0.5 \mu \mathrm{m}$ achieved at the unique infrared beamline of the Synchrotron Radiation Center, Wisconsin). Now, however, it is possible to configure a benchtop system for enhanced magnification, reducing pixel resolution from $5.5 \mu \mathrm{m}$ to $1.1 \mu \mathrm{m}$ using the sample objective, allowing for sub-cellular imaging possibilities [11].

In addition, there are now even more incentives to drive IR as a diagnostic tool due to the introduction of discrete frequency infrared (DFIR) imaging. The conventional thermal source is replaced with a high-intensity tuneable laser that has a customisable frequency range. This new IR technique provides the opportunity to collecting a handful of salient frequencies or discrete data ranges of discriminatory interest, thus creating a significant reduction in spectral acquisition time, with no SNR penalty [12]. By the combination of a multi-culture cell assay and DFIR technology, a preclinical high-throughput IR tool for objective classification of potent novel anticancer agents can now be realised. 


\section{References}

[1] R.H. Shoemaker, The NCl60 human tumour cell line anticancer drug screen, Nat. Rev. Cancer, 6 (2006) 813-823.

[2] S. Gaudenzi, D. Pozzi, P. Toro, I. Silvestri, S. Morrone, A. Congiu Castellano, Cell apoptosis specific marker found by Fourier Transform Infrared Spectroscopy, Spectroscopy, 18 (2004) 415-422.

[3] P. Bassan, A. Sachdeva, A. Kohler, C. Hughes, A. Henderson, J. Boyle, J.H. Shanks, M. Brown, N.W. Clarke, P. Gardner, FTIR microscopy of biological cells and tissue: Data analysis using resonant Mie scattering (RMieS) EMSC algorithm, Analyst, 137 (2012) 1370-1377.

[4] M.J. Baker, J. Trevisan, P. Bassan, R. Bhargava, H.J. Butler, K.M. Dorling, P.R. Fielden, S.W. Fogarty, N.J. Fullwood, K.A. Heys, C. Hughes, P. Lasch, P.L. Martin-Hirsch, B. Obinaju, G.D. Sockalingum, J. Sulé-Suso, R.J. Strong, M.J. Walsh, B.R. Wood, P. Gardner, F.L. Martin, Using Fourier transform IR spectroscopy to analyze biological materials, Nat. Protoc., 9 (2014) 1771-1791. [5] M. Jimenez-Hernandez, C. Hughes, P. Bassan, F. Ball, M.D. Brown, N.W. Clarke, P. Gardner, Exploring the spectroscopic differences of Caki-2 cells progressing through the cell cycle while proliferating in vitro, Analyst, 138 (2013) 3957-3966.

[6] D.R. Whelan, K.R. Bambery, L. Puskar, D. McNaughton, B.R. Wood, Synchrotron Fourier transform infrared (FTIR) analysis of single living cells progressing through the cell cycle, Analyst, 138 (2013) 3891-3899.

[7] A. Derenne, M. Verdonck, E. Goormaghtigh, The effect of anticancer drugs on seven cell lines monitored by FTIR spectroscopy, Analyst, 137 (2012) 3255-3264.

[8] F. Draux, P. Jeannesson, C. Gobinet, J. Sule-Suso, J. Pijanka, C. Sandt, P. Dumas, M. Manfait, G.D. Sockalingum, IR spectroscopy reveals effect of non-cytotoxic doses of anti-tumour drug on cancer cells, Anal. Bioanal. Chem., 395 (2009) 2293-2301.

[9] A.V. Rutter, M.R. Siddique, J. Filik, C. Sandt, P. Dumas, G. Cinque, G.D. Sockalingum, Y. Yang, J. Sulé-Suso, Study of gemcitabine-sensitive/resistant cancer cells by cell cloning and synchrotron FTIR microspectroscopy, Cytometry Part A, 85 (2014) 688-697.

[10] K.M. Dorling, M.J. Baker, Rapid FTIR chemical imaging: Highlighting FPA detectors, Trends Biotechnol., 31 (2013) 437-438.

[11] C. Hughes, A. Henderson, M. Kansiz, K.M. Dorling, M. Jimenez-Hernandez, M.D. Brown, N.W. Clarke, P. Gardner, Enhanced FTIR bench-top imaging of single biological cells, Analyst, (2015).

[12] Graeme Clemens, Benjamin Bird, Miles Weida, Jeremy Rowlette, M.J. Baker, Quantum cascade laser-based mid-infrared spectrochemical imaging of tissues and biofluids, SpectroscopyEurope, 26 (2014) 14-19. 
Figure

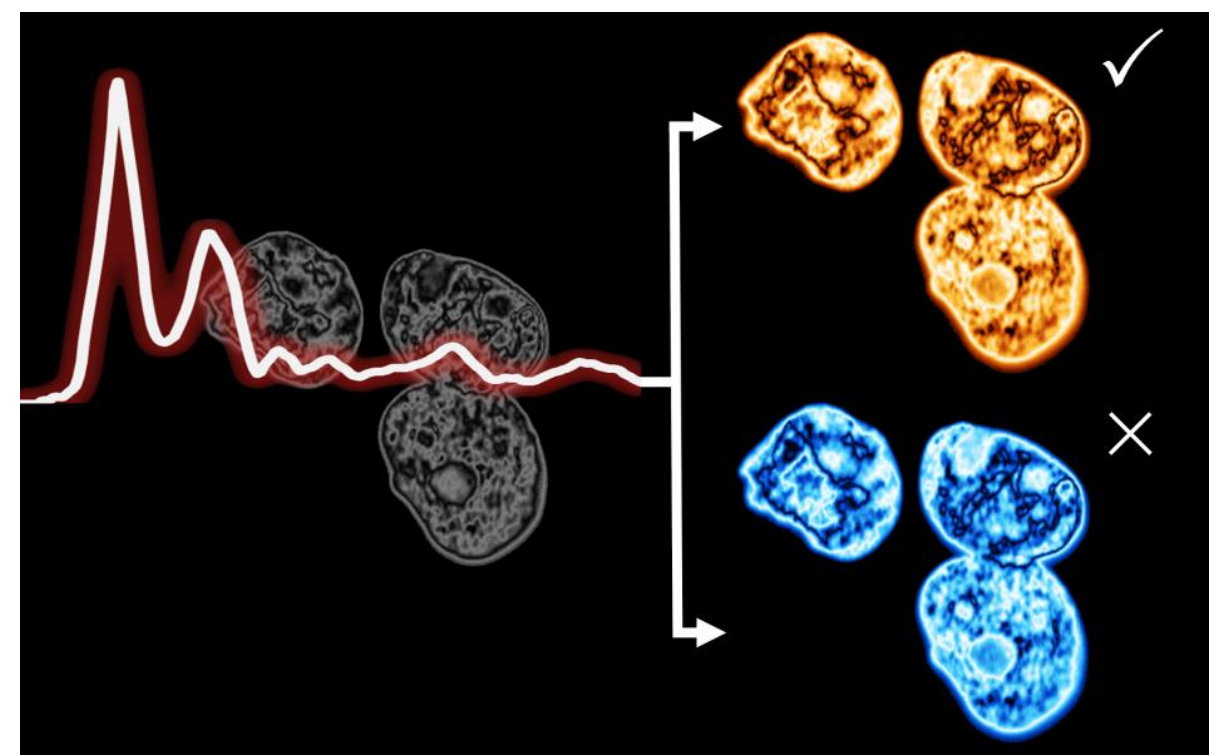

Figure 1: Infrared micro-spectroscopy can probe the global biochemical cellular fingerprint in a stainfree and non-destructive process, revealing spectral biomarkers of cytotoxic efficacy in drug-cell interaction assays. Recent developments in technology have afforded the power to quickly provide this information, thus highlighting the technique as a potential preclinical tool in the discovery process of potent novel chemotherapeutics. 